\title{
Performance of Long-Reach Passive Access Networks Using Injection-Locked Fabry-Perot Laser Diodes With Finite Front-Facet Reflectivities
}

\author{
Chien-Hung Yeh, Member, IEEE, Chi-Wai Chow, Senior Member, IEEE, Yu-Fu Wu, Sung-Ping Huang, \\ Yen-Liang Liu, and Ci-Ling Pan, Fellow, IEEE
}

\begin{abstract}
In this study, we use the injection-locked Fabry-Perot laser diodes (FP-LDs) with front-facet reflectivities of $10 \%$ and $35 \%$ respectively, as colorless optical networking unit (ONU) in long-reach passive optical networks (LR-PONs). Here, the injection-locked FP-LD is directly modulated at $2.5 \mathrm{Gbit} / \mathrm{s}$ on-off keying (OOK) and $10 \mathrm{Gbit} / \mathrm{s}$ orthogonal-frequency-division-multiplexing (OFDM) modulations for upstream traffic transmission over $60 \mathrm{~km}$ of single-mode fiber (SMF). Different operating parameters for the injection-locked FP-LD to achieve higher data rate transmission are reported.
\end{abstract}

Index Terms-Fabry-Perot Laser Diode (FP-LD), injectionlocked, long-reach, orthogonal-frequency-division-multiplexing (OFDM), passive optical network (PON).

\section{INTRODUCTION}

$\mathbf{R}$ ECENTLY, passive optical networks (PONs) are widely considered for different access network architectures, including the wireless and wired networks [1], [2]. Besides, various multiplexing methods, such as the time-division-multiplexing (TDM), orthogonal-frequency-division-multiplexing (OFDM), and wavelength-division-multiplexing (WDM) can be used to share the bandwidth in PONs. Furthermore, to meet the bandwidth demand of the next generation optical access network, WDM-PON is considered as a promising access solution due to its high capacity [3]. In WDM-PON, colorless transmitters [ref] are essential. Such transmitters are typically based on spectrum-sliced seeding for injection-locked Fabry-Perot lasers (FP-LD) [4], [5] or external-injection of reflective semiconductor optical amplifier (RSOA) [5], [7]. Currently, the transmission traffic rate of these solutions is

Manuscript received January 04, 2013; revised March 01, 2013; accepted March 19, 2013. Date of publication April 03, 2013; date of current version May 24, 2013.

C.-H. Yeh is with the Information and Communications Research Laboratories, Industrial Technology Research Institute (ITRI), Hsinchu 31040, Taiwan, and also with the Graduate Institute of Applied Science and Engineering, Fu-Jen Catholic University, New Taipei 24205, Taiwan.

C.-W. Chow, Y.-F. Wu, and S.-P. Huang are with the Department of Photonics and Institute of Electro-Optical Engineering, National Chiao Tung University, Hsinchu 30010, Taiwan (e-mail: cwchow@faculty.nctu.edu.tw).

Y.-L. Liu is with the Information and Communications Research Laboratories, Industrial Technology Research Institute (ITRI), Hsinchu 31040, Taiwan.

C.-L. Pan is with the Department of Physics and Institute of Photonics Technologies, National Tsing Hua University, Hsinchu 30013, Taiwan.

Color versions of one or more of the figures in this paper are available online at http://ieeexplore.ieee.org.

Digital Object Identifier 10.1109/JLT.2013.2254700 limited to $1.25 \mathrm{Gbit} / \mathrm{s}$ due to the high intensity noise of spectrum-sliced signal [8].

To effectively increase the modulation data rate and reduce the cost, several groups [9]-[11] proposed and demonstrated the use of optical OFDM-quadrature amplitude modulation (QAM) in carrier-distributed PONs. Moreover, hybrid WDM-TDM PON has been considered as a potential solution for next generation access networks. Due to the bandwidth-sharing of the TDM-PON, hybrid WDM-TDM PON would also provide a relative lower per-subscriber cost than pure WDM-PON by dividing a single wavelength to multiple subscribers, while still maintaining a relatively high per-subscriber bandwidth [12].

In order to reduce the total cost of PONs, one possible method is to simplify the fiber network architecture, and then the number of equipment interfaces and network devices can be reduced. A good example of such schemes is the long-reach (LR)-PONs [13], [14]. The LR-PON can combine metro and access networks into a single system. It has the benefit of high capacity and high split-ratio, and the transmission can be more than 40 $\mathrm{km}$ and up to $100 \mathrm{~km}[15]$.

In this work, we experimentally study $2.5 \mathrm{Gbit} / \mathrm{s}$ on-off keying (OOK) and $10 \mathrm{Gbit} / \mathrm{s}$ 16-QAM OFDM modulation upstream signals by using injection-locked FP-LD with $10 \%$ and $35 \%$ front-facet reflectivities, respectively, in a $60 \mathrm{~km}$ single-mode fiber (SMF) LR-PON system. The system characteristics of optical networking units (ONUs) at different $\mathrm{CW}$ injection powers, e.g., bit error rate (BER), side-mode suppression ratio (SMSR), and fiber transmission length are also analyzed.

\section{EXPERIMENT, RESULTS AND DISCUSSION}

Fig. 1 shows the experimental setup of the injection-locked FP-LD providing the upstream signal in each ONU. Here, output of the tunable laser source (TLS) was sent through an optical circulator $(\mathrm{OC})$ and a $1 \times 4$ array waveguide grating (AWG), and then launched into the FP-LD for injection-locking. In the experiment, the AWG was a Gaussian-shaped optical filter having a $3 \mathrm{~dB}$ bandwidth of $0.4 \mathrm{~nm}$. Its insertion loss was about $4 \mathrm{~dB}$. As shown in Fig. 1, the polarization controller (PC) was utilized to control the polarization state and obtain maximum output power of the FP-LD-based upstream signal. Then, the upstream signal of the injection-locked FP-LD was passed through the OC and single-mode fiber (SMF) of 60 $\mathrm{km}$, before being detected by a $2.5 \mathrm{GHz}$ bandwidth PIN receiver (Rx). Besides, an optical pre-amplifier was also used 


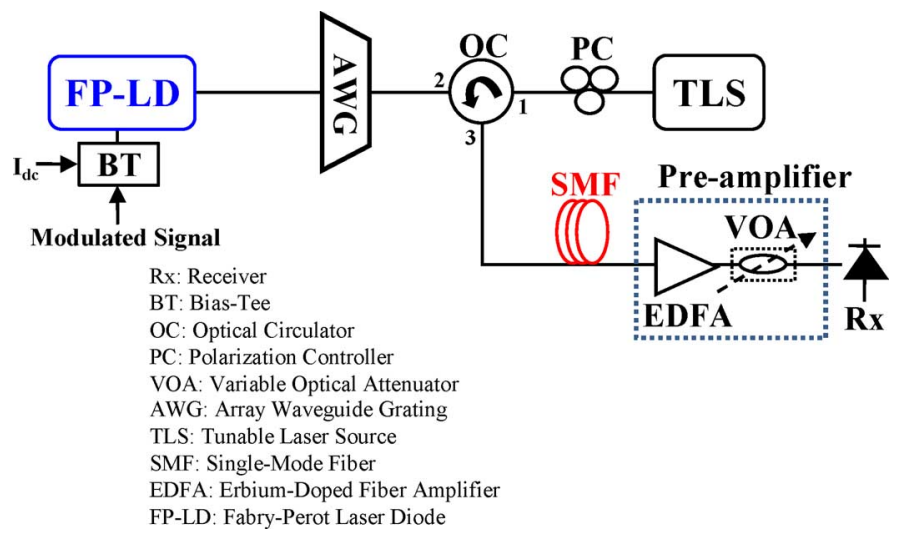

Fig. 1. Experimental setup of an injection-locked FP-LD acting as upstream signal in each ONU.

to enhance signal sensitivity. The pre-amplifier consisted of an erbium-doped fiber amplifier (EDFA); having $21 \mathrm{dBm}$ saturated power and $\sim 5 \mathrm{~dB}$ noise figure, and a variable optical attenuator (VOA). The optical spectrum and output power of proposed laser scheme were measured by an optical spectrum analyzer (OSA) with a $0.05 \mathrm{~nm}$ resolution and power meter (PM) respectively, respectively.

In this experiment, we employed two FP-LDs with frontfacet reflectivities of $35 \%$ and $10 \%$, respectively, in the proposed injection-locked FP-LD scheme. Fig. 2 shows the output power versus bias current (L-I) curves of the two FP-LDs. Their threshold currents were 10 and $16 \mathrm{~mA}$, respectively. antireflection (AR) coating was applied to make the front-facet reflectivity of the FP-LD to be $10 \%$. Such the AR coating allows much lower injection power for successful injection locking (we will discuss later in the paper), which also reduces undesirable backward reflection at the front-facet. As discussed in [16], reducing the front-facet reflectivity increases the optical injection wavelength locking range. Hence, the wavelength accuracy for the injection signal is less crucial for achieving injection locking in data transmission. Besides, the low front-facet reflectivity reduces the optical coherence of the FP-LD [17], the lasing of the FP-LD becomes more difficult, which could be indicated by the increase in threshold current as shown in Fig. 2. When biased at $35 \mathrm{~mA}$, the measured output powers of the two FP-LDs were 2.92 and $1.84 \mathrm{~mW}$, respectively. In order to achieve the same total output power (around $1.75 \mathrm{~mW}$ ), the operating currents of the two FP-LDs were set at 25 and $34 \mathrm{~mA}$, respectively.

Then, we measured the frequency responses of the $35 \%$ and $10 \%$ front-facet reflectivity FP-LDs with and without optical injection as shown in Fig. 3(a) and (b) respectively. We can observe the $3-\mathrm{dB}$ response of the $35 \%$ and $10 \%$ front-facet reflectivity FP-LDs were $0.6 \mathrm{GHz}$ and $0.8 \mathrm{GHz}$ respectively. Due to the use of weak optical injection, the increase in frequency response of the FPLDs was not significant before and after optical injection. However, the slope of the frequency response of the $35 \%$ front-facet reflectivity FP-LD was steeper. At the bandwidth of $2.5 \mathrm{GHz}$, the normalized response of the $35 \%$ FP-LD was $-8 \mathrm{~dB}$, while the normalized response of the $10 \%$ FP-LD was $-6 \mathrm{~dB}$.

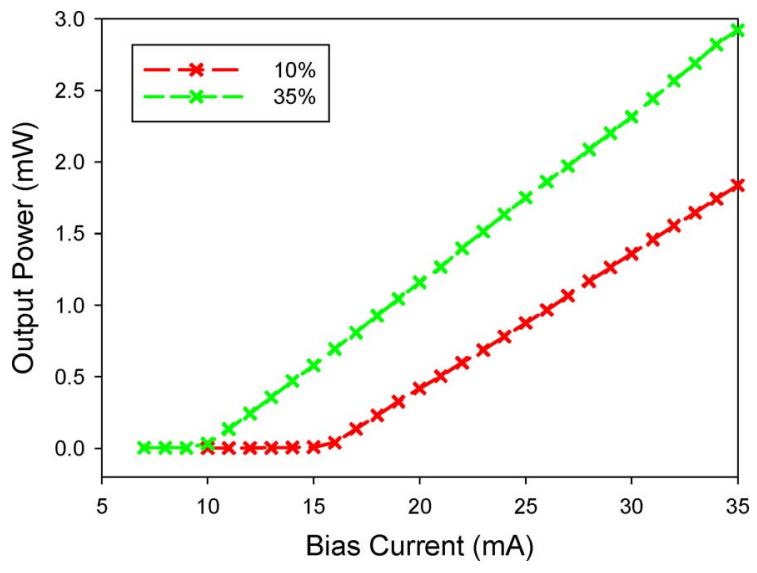

Fig. 2. The L-I curves of two FP-LDs with front-facet, reflectivities of $10 \%$ and $35 \%$.
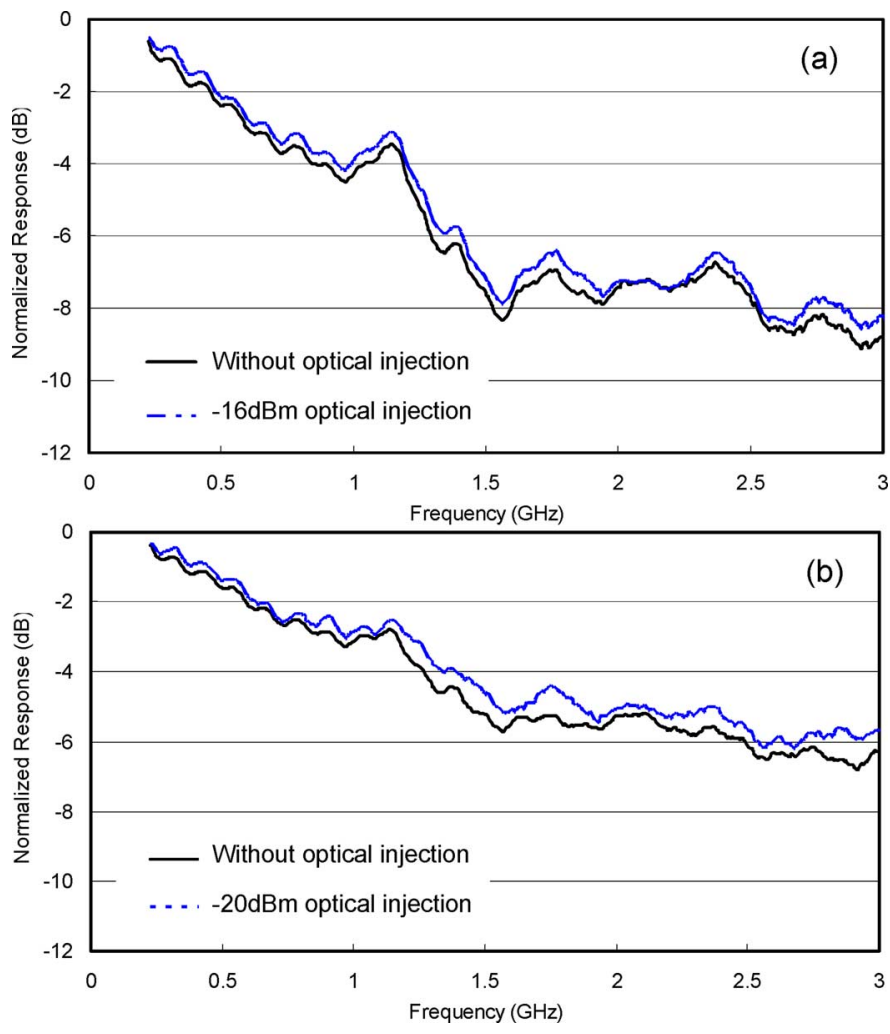

Fig. 3. Measured frequency responses of two FP-LDs with front-facet reflectivities of (a) $35 \%$ and (b) $10 \%$ with and without optical injection.

First of all, we performed the experiment using the proposed upstream signal with $35 \%$ front-facet reflectivity injection-locked FP-LD. Fig. 4(a) shows the output spectrum of FP-LD at the temperature of $25^{\circ} \mathrm{C}$, biased at $25 \mathrm{~mA}$ and without optical-injection. Its central wavelength was 1543.55 $\mathrm{nm}$ with a spectral power of $-8.6 \mathrm{dBm}$ and a mode-spacing of $1.1 \mathrm{~nm}$. To achieve $>30 \mathrm{~dB}$ side-mode suppression ratio (SMSR) for better upstream performance, various injection powers were used to launch into each output mode of original FP-LD for each injection wavelengths, as shown in Fig. 4(b). As expected, the smaller the spectral power of the lasing mode, a larger injection power it requires. Hence, to achieve the 

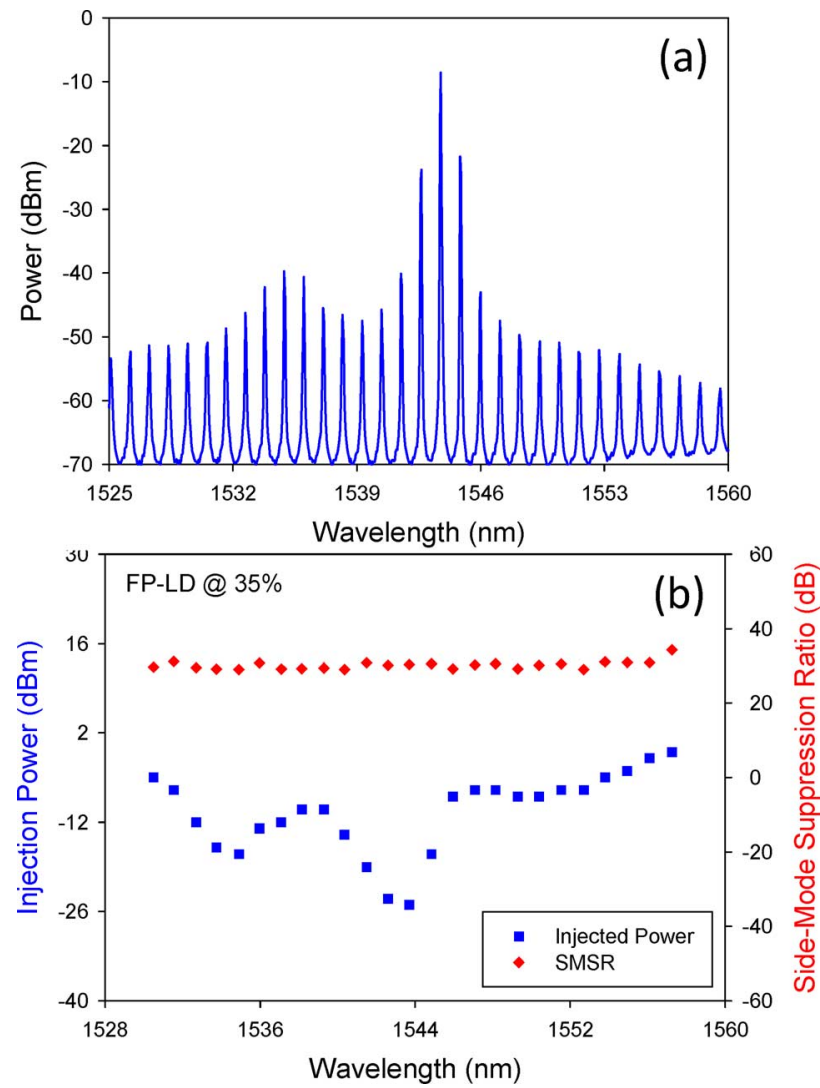

Fig. 4. (a) Output spectrum of the FP-LD without optical-injection, biased at $25 \mathrm{~mA}$ and at the temperature of $25^{\circ} \mathrm{C}$. (b) The wavelength dependence of injection power needed to achieve the same SMSR of around $30 \mathrm{~dB}$.

SMSR of $>30 \mathrm{~dB}$, the minimum injection powers required was between -25 and $-1 \mathrm{dBm}$ in the wavelengths range from 1530.50 to $1557.24 \mathrm{~nm}$ (see Fig. 4(b)).

With the injection wavelength set at $1543.55 \mathrm{~nm}$ launching into FP-LD for injection-locking, we plotted the output powers and SMSRs of the FP-LD at different injection powers in Fig. 5. The inset shows the output spectrum of injection-locked FP-LD at an injection power of $-16 \mathrm{dBm}$. Its corresponding output power and SMSR were $-7.8 \mathrm{dBm}$ and $31.6 \mathrm{~dB}$, respectively. Here, the measured output power of the injection-locked FP-LD was between -6.2 and $-7.9 \mathrm{dBm}$, while the injection power range was from -25 to $0 \mathrm{dBm}$. Besides, as can be seen in Fig. 5, no matter how we changed the injection power for the injectionlocked FP-LD within the injection range, the maximum power variation of injection-locked FP-LD was within $1.7 \mathrm{~dB}$ of its average value. This was due to the gain saturation effect of the injection-locked FP-LD. On the other hand, by increasing of injection power gradually, we could achieve a better SMSR.

To achieve long-reach fiber transmission over $60 \mathrm{~km}$ of SMF, an injection power of $-16 \mathrm{dBm}$ at $1543.55 \mathrm{~nm}$ was required for the injection-locked FP-LD, which can be directly modulated at $2.5 \mathrm{Gbit} / \mathrm{s}$ with non-return-to-zero (NRZ) pseudo random binary sequence (PRBS) of which the pattern length was $2^{31}-1$ to generate the upstream optical OOK signal. Fig. 6 presents the BER measurements of injection-locked FP-LD at the back-to-back (B2B) status and $60 \mathrm{~km}$ fiber transmission. The Rx sensitivities of -24.3 and $-23.6 \mathrm{dBm}$ were observed at the BER of $10^{-9}$

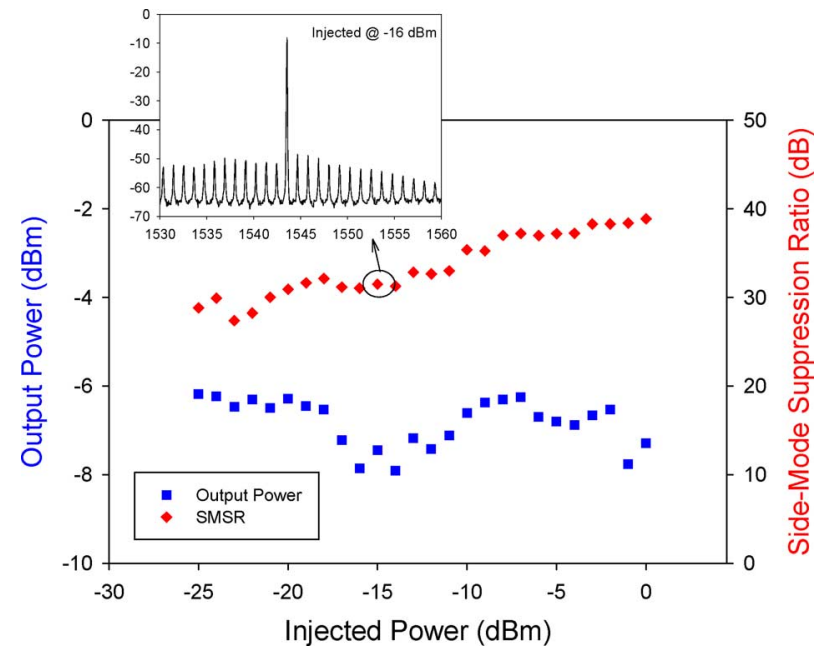

Fig. 5. Output powers and SMSRs of injection-locked FP-LD versus injection powers when the injection mode is set at $1543.55 \mathrm{~nm}$. The inset shows the output spectrum of injection-locked FP-LD.

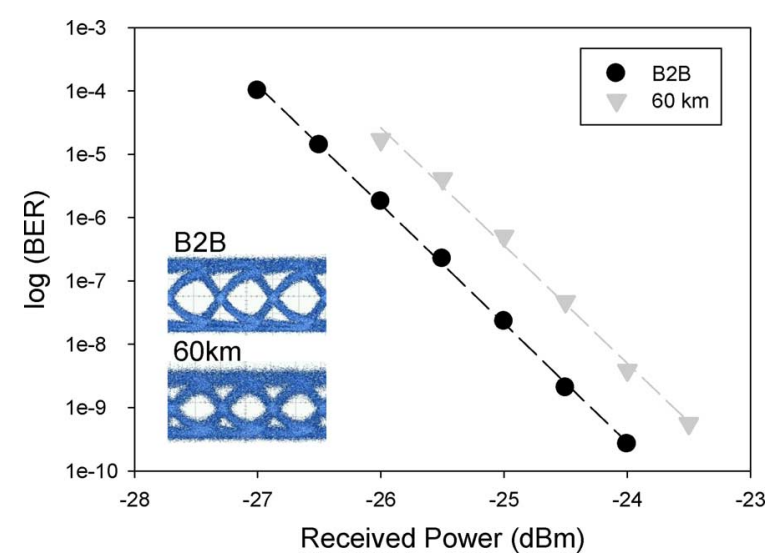

Fig. 6. BER performances of injection-locked FP-LD with $35 \%$ front-facet reflectivity at the B2B status and $60 \mathrm{~km}$ fiber transmission. Insets are the corresponding eye diagrams.

for the signal with and without the $60 \mathrm{~km}$ fiber transmission respectively. Hence, $<0.7 \mathrm{~dB}$ power penalty was measured at the BER of $10^{-9}$ after $60 \mathrm{~km}$ SMF transmission. However, in the same measurement, since the $\mathrm{CW}$ injection power was set to $-17 \mathrm{dBm}$, the measured BER can only achieve around $10^{-7}$ after $60 \mathrm{~km}$ fiber transmission. As a result, to achieve long-reach $60 \mathrm{~km}$ SMF transmission, the minimum injection power of $-16 \mathrm{dBm}$ must be employed for the injection-locked FP-LD laser scheme.

Next, we investigated the related characteristics of injectionlocked FP-LD with $10 \%$ front-facet reflectivity in PON system. In the measurement, the FP-LD was operated at $34 \mathrm{~mA}$ at the temperature of $25^{\circ} \mathrm{C}$. Thus, Fig. 7(a) shows the output spectrum of FP-LD without optical-injection, and its total output power was also obtained around $1.76 \mathrm{~mW}$. Here, the center wavelength of FP-LD was $1540.02 \mathrm{~nm}$ with a peak power of $-17.9 \mathrm{dBm}$, and its mode-spacing was measured at $0.8 \mathrm{~nm}$. Compared with Fig. 4(a), the maximum peak power (center wavelength) of $10 \%$ reflectivity FL-LD was less than that of 35\% reflectivity. So, in order to achieve a SMSR of $>30 \mathrm{~dB}$ in injection-locked FP-LD, the different injection powers were utilized to launch into each 

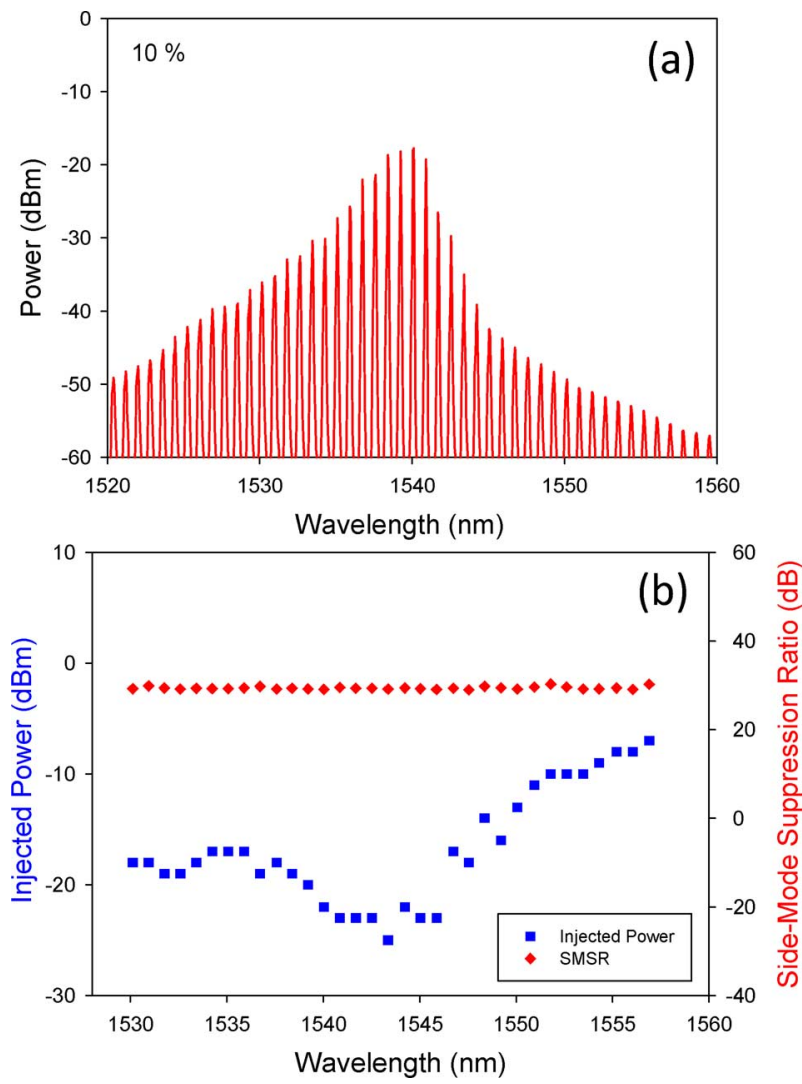

Fig. 7. (a) Output spectrum of the FP-LD without optical-injection, biased at $34 \mathrm{~mA}$ and at the temperature of $25^{\circ} \mathrm{C}$. (b) The wavelength dependence of injection power needed to achieve the same SMSR of around $30 \mathrm{~dB}(\mathrm{~b})$ The relationship of injection power and wavelength with the same SMSR of around $30 \mathrm{~dB}$.

output mode of FP-LD at the different injection wavelengths, as shown in Fig. 7(b). In the experiment, the required injection power was between -25 and $-7 \mathrm{dBm}$ in the wavelengths of 1530.50 to $1557.24 \mathrm{~nm}$, as illustrated in Fig. 7(b). By comparing Figs. 4(b) and 7(b), it was observed that the lower front-facet reflectivity FP-LD only required smaller $\mathrm{CW}$ injection power for effective injection-locking.

In the experiment, we chose an injection wavelength at $1540.02 \mathrm{~nm}$ launching into FP-LD with $10 \%$ front-facet reflectivity for injection-locking operation. Fig. 8 presents the corresponding output powers and SMSRs under different $\mathrm{CW}$ injection powers. The inset is the output spectrum of injection-locked FP-LD, and an injection power was set at $-20 \mathrm{dBm}$. Its output power and SMSR of $-9.8 \mathrm{dBm}$ and $18.1 \mathrm{~dB}$ were also observed. Moreover, the measured output powers of injection-locked FP-LD were between -7.5 and $-11.0 \mathrm{dBm}$ in the injection power range of -25 to $0 \mathrm{dBm}$. When the injection power increased gradually, the retrieved output power and SMSR of injection-locked FP-LD were also increased, as seen in Fig. 8. Besides, the observed SMSR was between 5.3 and $41.9 \mathrm{~dB}$ in the injection power range.

In order to achieve $60 \mathrm{~km}$ long-reach SMF transmission, the $1540.02 \mathrm{~nm} \mathrm{CW}$ injection wavelength with a $-20 \mathrm{dBm}$ power was used for the injection-locked FP-LD for upstream traffic. Here, the $1540.02 \mathrm{~nm}$ injection-locked FP-LD can be directly modulated at $2.5 \mathrm{Gbit} / \mathrm{s}$ with NRZ-PRBS format with a pattern

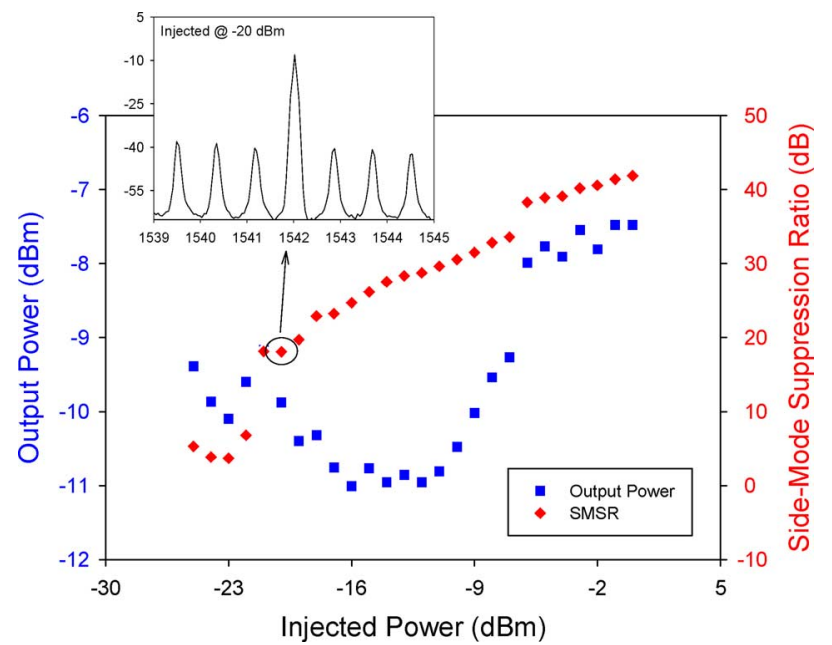

Fig. 8. Output powers and SMSRs of injection-locked FP-LD versus injection powers when the injection mode is set at $1540.02 \mathrm{~nm}$. The inset shows the output spectrum of injection-locked FP-LD.

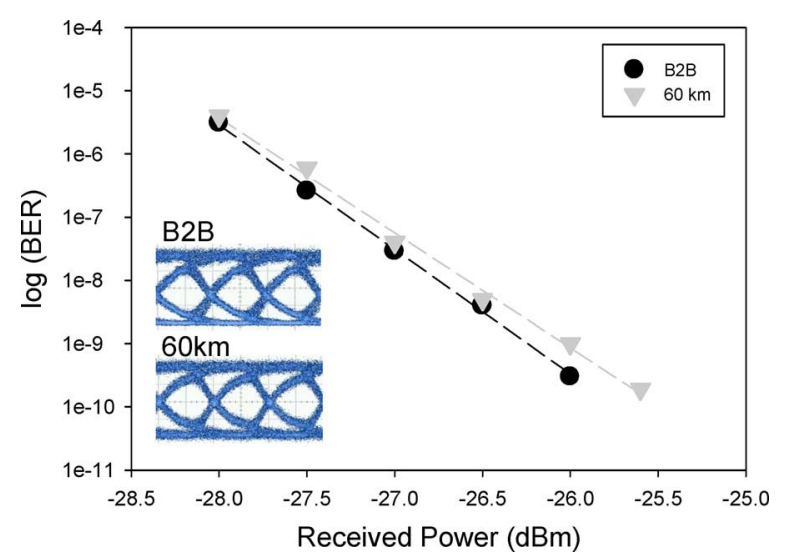

Fig. 9. BER performances of injection-locked FP-LD with $10 \%$ front-facet reflectivity at the B2B status and $60 \mathrm{~km}$ fiber transmission. Insets are the corresponding eye diagrams.

length of $2^{31}-1$ to produce the OOK upstream signal. Hence, Fig. 9 shows the BER performances of injection-locked FP-LD at the B2B status and $60 \mathrm{~km}$ fiber transmission. The Rx sensitivities of -26.1 and $-26.3 \mathrm{dBm}$ were obtained at the BER of $10^{-9}$ and nearly $0.2 \mathrm{~dB}$ power penalty is observed at the BER of $10^{-9}$ after $60 \mathrm{~km}$ fiber transmission. Besides, in the same measurement, when the $\mathrm{CW}$ injection power was set to $-21 \mathrm{dBm}$, the retrieved BER only achieved around $10^{-6}$ after $60 \mathrm{~km}$ fiber transmission. Under the $-21 \mathrm{dBm}$ injection power, the BER cannot achieve error free. Therefore, to achieve long-reach $60 \mathrm{~km} \mathrm{SMF}$ transmission, the minimum injection power of $-20 \mathrm{dBm}$ should be used in the injection-locked FP-LD with $10 \%$ front-facet reflectivity.

In order to increase the upstream traffic rate, optical OFDM-QAM modulation can be used for the injection-locked FP-LD. Here, the $10 \%$ front-facet reflectivity injection-locked FP-LD was used for the direct modulation of 16-QAM OFDM format to generate $10 \mathrm{Gbit} / \mathrm{s}$ upstream data rate. Here, the baseband electrical OFDM upstream signal was generated by an arbitrary waveform generator (AWG) using the Matlab ${ }^{\circledR}$ program. The signal processing of the OFDM transmitter 

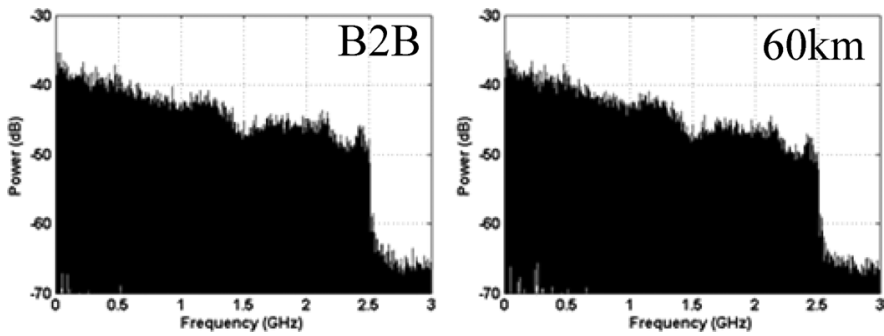

Fig. 10. Measured electrical spectra of the optical OFDM signal, when a $-13 \mathrm{dBm}$ injection power launches into the FP-LD, at the B2B state and after $60 \mathrm{~km}$ fiber transmission, respectively.

constructed by the serial-to-parallel conversion, QAM symbol encoding, inverse fast Fourier transform (IFFT), cyclic prefix (CP) insertion, and digital-to-analog conversion (DAC). 10 GSample/s sampling rate and 8 bit DAC resolution were set by the AWG, and CP of $1 / 64$ was used. Thus, 128 subcarriers of 16-QAM format occupied nearly $2.5 \mathrm{GHz}$ bandwidth from 0.0195 to $2.5195 \mathrm{GHz}$, with a fast-Fourier transform (FFT) size of 512. Here, $19.5 \mathrm{MHz}$ subcarrier spacing and $10 \mathrm{Gbit} / \mathrm{s}$ total data rate were achieved. Hence, the produced electrical 16-QAM OFDM signal can be applied to the FP-LD via a bias-tee (BT). Then the upstream signal was direct-detected via a $2.5 \mathrm{GHz}$ PIN Rx, and the received OFDM signal was captured by a real-time $50 \mathrm{GHz}$ sampling oscilloscope for signal demodulation. To demodulate the vector signal, the off-line DSP program was employed. Then the demodulation process contained the synchronization, FFT, one-tap equalization, and QAM symbol decoding. Finally, the BER can be calculated according to the observed signal-to-noise ratio (SNR).

Fig. 10(a) and (b) show the measured electrical spectra of the optical OFDM signal, when using $-13 \mathrm{dBm}$ injection power launching into the FP-LD, at the B2B state and after $60 \mathrm{~km}$ fiber transmission respectively. The FP-LD was dc-based properly. After $60 \mathrm{~km}$ fiber transmission, the electrical power would degrade in the higher frequency domain due to the RF fading and fiber chromatic dispersion.

To achieve the LR signal transmission, the $\mathrm{CW}$ injection power of $-14 \mathrm{dBm}$ was required for the injection-locked FP-LD. Hence, Fig. 11 presents the BER performances of 10 Gbit/s, 16-QAM OFDM upstream traffic at the B2B and after $60 \mathrm{~km}$ SMF transmission, respectively. The insets of Fig. 10 are the corresponding constellation diagrams, measuring at the forward error correction (FEC) threshold (SNR $=16.5 \mathrm{~dB}$ and $\mathrm{BER}=10^{-3}$ ), and the received power was set at $-16 \mathrm{dBm}$. Here, when the FEC was used, the received sensitivities were observed at -16.6 and $-14.6 \mathrm{dBm}$, under the B2B state and 60 $\mathrm{km}$ fiber transmission, respectively. As a result, the measured power penalty was $\sim 2.0 \mathrm{~dB}$, in the fiber transmission length of $60 \mathrm{~km}$. Moreover, when we reduced the injection power to $-14 \mathrm{dBm}$ for FP-LD in the same experiment, the measured BER cannot achieve FEC threshold level under $60 \mathrm{~km}$ fiber transmission. That is to say, the minimum $\mathrm{CW}$ injection power of $-15 \mathrm{dBm}$ was needed for the successful $10 \mathrm{Gbit} / \mathrm{s}$ 16-QAM OFDM signal transmission in an injection-locked FP-LD.

The direct modulation bandwidth of a free-run FP-LD is determined by the relaxation oscillation frequency $\left(\omega_{R O}\right)$ of the

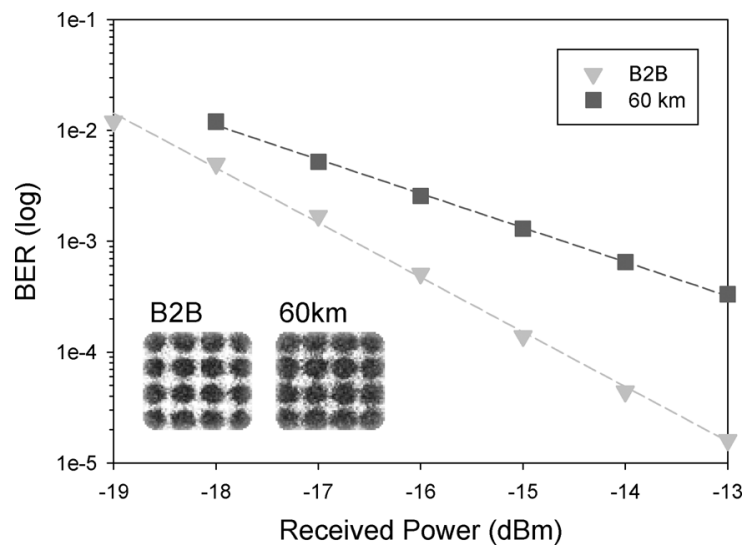

Fig. 11. BER performances of $10 \mathrm{Gbit} / \mathrm{s}$ 16-QAM OFDM upstream traffic at the B2B and after $60 \mathrm{~km}$ SMF transmission, respectively. The insets of Fig. 10 are the corresponding constellation diagrams.

FP-LD, which depends on the bias current of the FP-LD, photo lifetime [18]. Under optical injection locking, the enhanced relaxation oscillation frequency $\left(\omega_{R}\right)$ can be described in (1) [19]

$$
\omega_{R}^{2} \approx \omega_{R 0}^{2}+\kappa^{2}\left(\frac{A_{\mathrm{inj}}}{A_{0}}\right)^{2} \sin ^{2} \theta
$$

where $\kappa$ is the coupling coefficient, $\mathrm{A}_{\mathrm{inj}}$ and $\mathrm{A}_{0}$ are the amplitude of the injected signal and free-running slave laser signal respectively, $\theta$ is the steady-state phase difference between the injection-locked slave laser and the master laser. We can observe in [19] that increasing the optical injection ratio enhances the relaxation oscillation frequency; hence increasing the modulation speed of the injection-locked laser. Recent demonstrations show that the relaxation oscillation frequencies can be increased to $>10 \mathrm{GHz}$ using moderate power $\mathrm{CW}$ injection (injection power $-2.2 \mathrm{dBm}$ ) [20], and up to $72 \mathrm{GHz}$ using ultrahigh optical injection (injection power $+23 \mathrm{dBm}$ ) [21].

However, in an optical carrier distributed network, like the proposed WDM-PON in this paper, the CW optical light source is usually located in the remote node or at the central office far away. The CW optical power will be highly attenuated by the fiber splitter, AWG and SMF before injecting into the FP-LD at the user ONU; hence using high power optical injection locking is impractical in the WDM-PON. As a result, in this paper, we use weak optical injection powers of $-16 \mathrm{dBm}$ and $-20 \mathrm{dBm}$ for the FP-LDs with front-facet reflectivities of $35 \%$ and $10 \%$ respectively. It is expected that by increasing the injection optical power, higher bit-rate can be achieved for both types of FP-LDs.

\section{CONCLUSION}

We experimentally demonstrated $2.5 \mathrm{Gbit} / \mathrm{s}$ OOK and 10 Gbit/s 16-QAM OFDM modulation upstream signals using injection-locked FP-LD-based ONU with weak optical injection power in a LR-PON system. We can observe that lower front-facet reflectivity FP-LD required a smaller $\mathrm{CW}$ injection power for injection-locking. In the experiment, $-20 \mathrm{dBm}$ and $-16 \mathrm{dBm}$ of $\mathrm{CW}$ injection powers were used for the injection locking of the $10 \%$ and $35 \%$ front-facet reflectivity FP-LD respectively. 
Then, we analyzed the performance of $2.5 \mathrm{Gbit} / \mathrm{s}$ OOK and $10 \mathrm{Gbit} / \mathrm{s}$ OFDM modulation schemes using the same injected-locked $10 \%$ front-facet reflectivity FP-LD. Although using OFDM-QAM modulation in the injection-locked FP-LD can enhance the data rate by 4 times (from $2.5 \mathrm{Gbit} / \mathrm{s}$ to 10 Gbit/s), it sacrificed the Rx sensitivity, thus reducing the numbers of supported ONUs in the LR-PON. Besides, the power penalty was also increased when using OFDM modulation.

\section{REFERENCES}

[1] C. W. Chow, S. P. Huang, L. G. Yang, and C. H. Yeh, "Extendedreach access network with downstream radio-over-fiber (ROF) signal and upstream NRZ signal using orthogonal-WDM," Opt. Exp., vol. 20, no. 16, pp. 16757-16762, 2012.

[2] C. H. Yeh, C. W. Chow, and H. Y. Chen, "Simple colorless WDM-PON with Rayleigh backscattering noise circumvention Employing $m$-QAM OFDM downstream and remodulated OOK upstream signals," J. Lightw. Technol., vol. 30, no. 13, pp. 2151-2155, 2012.

[3] Y. C. Chi, C.-J. Lin, S.-Y. Lin, and G. R. Lin, "The reuse of downstream carrier data erased by self-feedback SOA for bidirectional DWDM-PON transmission," J. Lightw. Technol., vol. 30, no. 19, pp. 3096-3102, 2012

[4] J. H. Lee, K. Lee, Y.-G. Han, S. B. Lee, and C. H. Kim, "Single, depolarized, CW supercontinuum-based wavelength division multiplexed passive optical network architecture with C-band OLT, L-band ONU, and U-band monitoring," J. Lightw. Technol., vol. 25, no. 10, pp. 2891-2897, 2007.

[5] Q. T. Nguyen, P. Besnard, L. Bramerie, A. Shen, G. H. Duan, C. Kazmierski, and J.-C. Simon, "Bidirectional transmission in colourless WDM-PON based on injection-locked Fabry-Perot laser at $2.5 \mathrm{Gbit} / \mathrm{s}$ using low-cost seeding source," in Proc. ECOC, 2009, Paper 6.5.1.

[6] K. Y. Cho, Y. J. Lee, H. Y. Choi, A. Murakami, A. Agata, Y Takushima, and Y. C. Chung, "Effects of reflection in RSOA-based WDM PON utilizing remodulation technique," J. Lightw. Technol., vol. 27, no. 10, pp. 1286-1295, 2009.

[7] C. H. Yeh, C. W. Chow, Y. F. Wu, and H. Y. Chen, "Demonstrations of 10 and $40 \mathrm{Gbps}$ upstream transmissions using $1.2 \mathrm{GHz}$ RSOA-based ONU in long-reach access networks," Opt. Fiber Technol., vol. 18, no. 2, pp. 63-67, 2012

[8] G.-R. Lin, Y.-H. Lin, and Y.-C. Chang, "Theory and experiments of a mode beating noise suppressed and mutually injection-locked Fabry-Perot laser diode and erbium-doped fiber amplifier link," IEEE J. Quantum Electron., vol. 40, no. 8, pp. 1014-1022, 2004.
[9] Z. Cao, J. Yu, H. Zhou, W. Wang, M. Xia, J. Wang, Q. Tang, and L. Chen, "WDM-RoF-PON architecture for flexible wireless and wireline layout," J. Opt. Commun. Netw., vol. 2, no. 2, pp. 117-121, 2010.

[10] Y.-T. Hsueh, M.-F. Huang, S.-H. Fan, and G.-K. Chang, "Demonstration of converged bidirectional OFDM-m-QAM RoF and WDMOFDM-PON access networks," in Proc. of OFC, 2011, Paper OWK5.

[11] Y. -C. Chi, Y. -C. Li, H. -Y. Wang, P. -C. Peng, H. -H. Lu, and G. -R Lin, "Optical 16-QAM-52-OFDM transmission at $4 \mathrm{Gbit} / \mathrm{s}$ by directly modulating a coherently injection-locked colorless laser diode," Opt. Exp., vol. 20, pp. 20071-20077, 2012.

[12] C. W. Chow, C. H. Yeh, C. H. Wang, F. Y. Shih, and S. Chi, "Signal remodulation of OFDM-QAM for long reach carrier distributed passive optical networks," IEEE Photon. Technol. Lett., vol. 21, no. 11, pp. 715-717, 2009

[13] I. T. Monroy, F. Ohman, K. Yvind, R. Kjaer, C. Peucheret, A. M. J. Koonen, and P. Jeppesen, " $85 \mathrm{~km}$ long reach PON system using a reflective SOA-EA modulator and distributed Raman fiber amplification," in Proc. of LEOS Annu. Meeting, 2006, Paper WEE4.

[14] G. Talli and P. D. Townsend, "Hybrid DWDM-TDM long-reach PON for next-generation optical access," J. Lightw. Technol., vol. 24, no. 7, pp. 2827-2834, 2006.

[15] C. W. Chow and C. H. Yeh, "Mitigation of Rayleigh backscattering in $10-\mathrm{Gb} / \mathrm{s}$ downstream and 2.5-Gb/s upstream DWDM 100-km longreach PONs," Opt. Exp., vol. 19, no. 6, pp. 4970-4976, 2011.

[16] G. -R. Lin, Y. -S. Liao, Y. -C. Chi, H. -C. Kuo, G. -C. Lin, H. -L. Wang, and Y. -J. Chen, "Long-cavity Fabry-Perot laser amplifier transmitter with enhanced injection-locking bandwidth for WDM-PON application," J. Lightw. Technol., vol. 28, no. 20, pp. 2925-2932, 2010

[17] G.-R. Lin, H.-L. Wang, G.-C. Lin, Y.-H. Huang, Y.-H. Lin, and T.-K Cheng, "Comparison on injection-locked Fabry-Perot laser diode with front-facet reflectivity of $1 \%$ and $30 \%$ for optical data transmission in WDM-PON system," J. Lightw. Technol., vol. 27, no. 14, pp. 2779-2785, 2009

[18] G. P. Agrawal, Fiber-Optic Communication Systems. New York, NY, USA: Wiley.

[19] H. -K. Sung, E. K. Lau, and M. C. Wu, "Optical properties and modulation characteristics of ultra-strong injection-locked distributed feedback lasers," IEEE J. Sel. Top. Quantum Electron., vol. 13, no. 5, pp. 1215-1221, 2007.

[20] Z. Xu, Y. J. Wen, W. -D. Zhong, C. -J. Chae, X. -F. Cheng, Y. Wang, C. Lu, and J. Shankar, "High-speed WDM-PON using CW injection-locked Fabry-Pérot laser diodes," Opt. Exp., vol. 15, no. 6, pp. 2953-2962, 2007.

[21] E. K. Lau, H. K. Sung, and M. C. Wu, "Ultra-high, 72 GHz resonance frequency and $44 \mathrm{GHz}$ bandwidth of injection-locked 1.55- $\mu \mathrm{m} \mathrm{DFB}$ lasers," in Proc. OFC, 2006, OTHG2.

Author biographies not included by author request due to space constraints. 